\title{
Short-term Hemodynamic Variability in Supine and Tilted Position in Young Women
}

\author{
G Cybulski1,2, E Koźluk ${ }^{3}$, A Piątkowska ${ }^{3}$ E Michalak ${ }^{4}$, A Strasz ${ }^{5}$, \\ A Gąsiorowska ${ }^{2}$, W Niewiadomski ${ }^{2}$ \\ ${ }^{1}$ Institute of Metrology and Biomedical Engineering, WUT, Warsaw, Poland \\ ${ }^{2}$ Department of Applied Physiology, Mossakowski Medical Research Centre, PAS, Warsaw, Poland \\ ${ }^{3}$ Second Chair and Department of Cardiology, Medical University of Warsaw, Warsaw, Poland \\ ${ }^{4}$ Cardinal Stefan Wyszynski Institute of Cardiology, Warsaw, Poland \\ ${ }^{5}$ Department of Experimental and Clinical Physiology, Medical University of Warsaw, Poland
}

\begin{abstract}
The aim of the study was to evaluate short-term changes in hemodynamic parameters observed in supine and tilted positions.

Six young women (age: 21-25) participated in the study. The cardiac inter-beat interval (RR), stroke volume $(S V)$, ejection time (ET) and pre-ejection period (PEP) parameters were followed over two six-minute periods, in supine position and 10 minutes after a 60-degree head-up tilting manoeuvre, using continuously recorded impedance cardiography (ICG) and electrocardiography (ECG) signals. Hemodynamic variability was evaluated using standard deviation (SD), coefficient of variation $(C V)$ and quartile deviation (QD).

For the supine position, the mean (M), $S D, C V$ and $Q D$ of the observed parameters were as follows. SV: $66 \mathrm{ml}$, $11 \mathrm{ml}, 17.5 \%, 16 \mathrm{ml}$. RR: $878 \mathrm{~ms}, 100 \mathrm{~ms}, 11.2 \%, 111$ ms. ET: 292 ms, 32 ms, 11.4 \%, 47 ms. PEP: 115 ms, 15 ms, $13.4 \%, 19$ ms.

In the tilted position, the following were observed. SV: $53 \mathrm{ml}, 10 \mathrm{ml}, 19.8 \%, 12.5 \mathrm{ml}$. RR: $736 \mathrm{~ms}, 88 \mathrm{~ms}, 11.8$ \%, 74 ms. ET: 252 ms, 30 ms, 11.7 \%, 23 ms. PEP: 134 ms, $12 \mathrm{~ms}, 9 \%, 13 \mathrm{~ms}$.

The changes in hemodynamic variability caused by tilting are not unidirectional.
\end{abstract}

\section{Introduction}

The analysis of cardiovascular system response to an orthostatic head-up tilt test may yield diagnostic data on autonomic control [1] and help to predict the occurrence of orthostatic syncope [2]. Cardiac rhythm variability has been intensively studied in supine and other body positions in humans. However, limited data are available regarding fluctuations of hemodynamic parameters, especially during orthostatic tests. Cybulski et al. [3] analysed central hemodynamic variability in a small group of subjects who either had atrial fibrillation or were healthy. It was concluded that the variability of SV, estimated by the coefficient of variation, was mainly caused by changes in the amplitude of the signal and that variation in ET has a smaller effect. Siebert et al. [4] analysed stroke volume variability and heart rate power spectrum in relation to posture changes in healthy subjects. They concluded that the combined analysis of heart rate variability (HRV) and stroke volume variability (SVV) revealed different cardiovascular responses to postural stress in the three age groups considered. Beatto-beat variability of stroke volume output velocity (SVOV) at rest has also been measured in healthy subjects and in patients 10-14 days after acute myocardial infarction [5].

\subsection{Motivation}

The analysis of changes in stroke volume and systolic time intervals (including their variability) seems to be a potential source of information on coupling between the cardiovascular system and autonomic dysfunction or modification induced by several environmental and/or pathological factors [6,7]. Possibly, it could be used for: monitoring the effects of neurodegenerative processes [8], analysis of sleep apnoea disorders [9], monitoring the effectiveness of physical training and exercise [10] and predicting orthostatic intolerance [2].

\subsection{Purpose of study}

Thus, the aim of the study was to evaluate short-term variability in hemodynamic parameters - stroke volume (SV), ejection time (ET), pre-ejection period (PEP) observed in the supine and tilted positions in young, 
healthy female subjects.

\section{Material and methods}

\subsection{Subjects and procedure}

To estimate hemodynamic variability before and after the tilt test, we used data from our impedance cardiography signal database. These data were originally collected for a comparison of the results of hemodynamic parameters obtained from signals recorded simultaneously using two methods: ICG and pulse Doppler echocardiography. The result of that comparison was published earlier [11]. In this study. we used the signals obtained from six young, healthy female subjects (ages 21-25). The subjects remained recumbent for at least 15 minutes before examination. Impedance cardiography (ICG) and electrocardiographic (ECG) signals were recorded continuously in the supine position and following a 60-degree head-up tilt manoeuvre.

\subsection{Method and instrumentation}

We used a wearable ambulatory impedance cardiography recorder (Reomonitor), previously described [12-14]. This device was constructed for noninvasive acquisition of central hemodynamic data during everyday activity. The analogue part of Reomonitor consists of a one-channel ECG and a miniaturized impedance cardiograph. Changes in the thoracic impedance, reflecting the stroke volume (SV), were measured using the tetrapolar method. An alternating current of $100 \mathrm{kHz}$ (with a stable amplitude $<5 \mathrm{~mA}$ ) oscillated between application electrodes while the voltage (reflecting the impedance) was measured on the receiving electrodes. ECG and the first derivative of the impedance cardiography signal $(\mathrm{dz} / \mathrm{dt})$ were sampled (at $200 \mathrm{~Hz}$ ) with 8-bit resolution. Additionally, the basic impedance signal (Z0) was sampled every 5 seconds. Stroke volume was evaluated using the Kubicek formula [15]. The validation and reliability of impedance cardiography have been reviewed many times $[16,17]$. Ambulatory monitoring with the Reomonitor was verified using echocardiography in both the supine and tilted position [11].

\subsection{Statistical analysis}

Hemodynamic variability was evaluated using the standard deviation (SD), coefficient of variation (CV) and quartile deviation (QD) of the stroke volume derived from impedance cardiography (ICG), ejection time (ET) and pre-ejection period (PEP). Additionally, the intervals between cardiac beats (RR) were analysed. For each parameter in both supine and tilted position, the mean values (M) were calculated. QD was calculated as half of the difference between upper quartile (Q3) and lower quartile (Q1).

Analysis was performed for two six-minute periods, recorded in the supine position and 10 minutes after the tilting manoeuvre. Artefacts were removed from the analysis based on the clearly defined criteria for each variable.

\section{Results}

Table 1 contains the descriptive statistical parameters for SV. Tilting caused a decrease in the mean value of SV. Although CV increased, QD decreased.

Table 1. Descriptive characteristics of stroke volume (SV) in the supine and tilted positions.

\begin{tabular}{lll}
\hline Stroke volume (SV) & Supine & Tilted \\
\hline Mean [ml] & 66 & 53 \\
Standard deviation [ml] & 11 & 10 \\
Coeff. variation [\%] & 17.5 & 19.8 \\
Quartile deviation [ml] & 16 & 12.5 \\
\hline
\end{tabular}

Table 2 contains the same parameters for ET. Tilting caused a decrease in the mean value of ET. Although SD and CV were not significantly modified, QD decreased.

Table 2. Descriptive characteristic of ejection time (ET) in supine and tilted positions.

\begin{tabular}{lll}
\hline Ejection time (ET) & Supine & Tilted \\
\hline Mean [ms] & 292 & 252 \\
Standard deviation [ms] & 32 & 30 \\
Coeff. variation [\%] & 11.4 & 11.7 \\
Quartile deviation [ms] & 47 & 23 \\
\hline
\end{tabular}

Table 3 contains the descriptive statistical parameters for PEP. Tilting caused an increase in the mean value of PEP. It was observed that SD, CV and QD decreased.

Table 3. Descriptive characteristic of pre-ejection period (PEP) in supine and tilted positions.

\begin{tabular}{lll}
\hline Pre-ejection period (PEP) & Supine & Tilted \\
\hline Mean [ms] & 115 & 134 \\
Standard deviation [ms] & 15 & 12 \\
Coeff. variation [\%] & 13.4 & 9.0 \\
Quartile deviation [ms] & 19 & 13 \\
\hline
\end{tabular}

Table 4 contains the descriptive statistics for the amplitude (AMP) of dz/dtmax. Tilting caused no significant increase in the mean value of AMP, SD and QD. CV did not change significantly. 
Table 4. Descriptive characteristic of the amplitude of $\mathrm{dz} / \mathrm{dtmax}$ (AMP).

\begin{tabular}{lll}
\hline Amplitude (AMP) & Supine & Tilted \\
\hline Mean $[\Omega / \mathrm{s}]$ & 1.95 & 2.2 \\
Standard deviation $[\Omega / \mathrm{s}]$ & 0.26 & 0.30 \\
Coeff. variation $[\%]$ & 13.6 & 13.7 \\
Quartile deviation $[\Omega / \mathrm{s}]$ & 0.31 & 0.38 \\
\hline
\end{tabular}

Table 5 contains the statistics for RR. Tilting caused the mean value of RR to decrease, along with $\mathrm{SD}$ and QD. No change in CV was observed.

Table 5. Descriptive characteristic of RR intervals (RR).

\begin{tabular}{lll}
\hline RR intervals & Supine & Tilted \\
\hline Mean [ms] & 878 & 736 \\
Standard deviation [ms] & 100 & 88 \\
Coeff. variation [\%] & 11.2 & 11.8 \\
Quartile deviation [ms] & 111 & 74 \\
\hline
\end{tabular}

\section{Discussion and conclusions}

The advantages/disadvantages and application perspectives of the ICG method have been presented in review publications $[14,18,19]$. Fellahi and Fisher [19] noted that, despite some disappointing results for the measurement of absolute values of cardiac output in comparison with some reference methods. ICG might be "interesting in guiding the spontaneous or induced changes in cardiac output and medical decision-making at the bedside”. In a monograph, Cybulski [14] reported the usefulness of ambulatory ICG in the non-invasive assessment of hemodynamic impairment caused by cardiac arrhythmias, verification of VVI pacemakers and optimisation of AV delay in dual-chamber pacing systems during normal daily activity. It was also pointed out that an ambulatory version of ICG could be used to record transient events, which would be difficult or even impossible to visualise using other, well established, "classical" methods. Some perspectives of ICG development were presented in Cybulski et al. [18].

The main research limitation of the study is the small size of the sample. Another is associated with uncertainty of the method, regarding the limitations of the proper detection of the B-point on the dz/dt curve in ICG [20]. This results in uncertainty in determination of systolic time intervals (ET, PEP, and their derivatives). We feel unable to estimate the impact of variation in systolic time intervals caused by method uncertainties in the variation induced by physiological changes. We could only assume that B-detection uncertainty is constant with respect to body position.

These preliminary results show that relative measures of hemodynamic parameter variability after tilting were different compared to those observed in the supine position; the observed changes were not unidirectional.

\section{Acknowledgements}

The study was supported by the research programs of institutions the authors are affiliated with.

\section{References}

[1] Cybulski G. Influence of age on the immediate cardiovascular response to the orthostatic maneouvre. European Journal of Applied Physiology 1996;73:563-72.

[2] Koźluk E, Cybulski G, Piątkowska A, Zastawna I, Niewiadomski W, Strasz A, Gąsiorowska A, Kempa M, Kozłowski D, Opolski G. Early hemodynamic response to the tilt test in patients with syncope. Arch Med Sci 2014;10(6):1078-85.

[3] Cybulski G, Ziółkowska E, Książkiewicz A, Łukasik W, Niewiadomski W, Kodrzycka A, Pałko T. Application of Impedance Cardiography Ambulatory Monitoring Device for Analysis of Central Hemodynamics Variability in Atrial Fibrillation. Computers in Cardiology 1999;26:563-6.

[4] Siebert J, Drabik P, Lango R, Szyndler K. Stroke volume variability and heart rate power spectrum in relation to posture changes in healthy subjects. Med Sci Monitor 2004; 10(2):MT31-7.

[5] Zubarev MA, Schekotov VV, Parandey OR, Dumler AA. Beat - to - beat variability of stroke volume output velocity measured by an impedance cardiographic method. IFMBE Proceedings 2007;17:579-81

[6] Toska K, Eriksen M. Respiration-synchronous fluctuations in stroke volume, heart rate and arterial pressure in humans. J Physiol 1993;472:501-12.

[7] Niizeki K, Saitoh T. Analysis of cardiorespiratory phase coupling and cardiovascular autonomic responses during food ingestion. Physiol Behav 2016;159:1-13.

[8] Marongiu E, Olla S, Magnani S, Palazzolo G, Sanna I, Tocco F, Marcelli M, Loi A, Corona F, Mulliri G, Concu A, Crisafulli A. Metaboreflex activity in multiple sclerosis patients. European Journal of Applied Physiology 2015;115(12):2481-90.

[9] Ahmad S, Batkin I, Kelly O, Dajani HR, Bolic M, Groza V. Multiparameter Physiological Analysis in Obstructive Sleep Apnea Simulated With Mueller Maneuver. IEEE Trans Instrumentation and Measurement 2013;62(10):275162.

[10] Gratze G, Mayer H, Luft FC, Skrabal F. Determinants of fast marathon performance: low basal sympathetic drive, enhanced postcompetition vasodilatation and preserved cardiac performance after competition. $\mathrm{Br} \mathrm{J}$ Sports Med 2008;42(11):882-8

[11] Cybulski G, Michalak E, Koźluk E, Piątkowska A, Niewiadomski W. Stroke volume and systolic time intervals: beat-to-beat comparison between echocardiography and ambulatory impedance cardiography in supine and tilted positions. Medical and Biological Engineering and Computing 2004;42:707-11.

[12] Cybulski G, Książkiewicz A, Łukasik W, Niewiadomski 
W, Pałko T. Ambulatory monitoring device for central hemodynamics and ECG signals recording on PCMCIA flash memory cards. Computers in Cardiology 1995;22:505-7.

[13] Cybulski G. Ambulatory impedance cardiography: new possibilities (Letter to the Editor). J Appl Physiol 2000;88:1509-10.

[14] Cybulski G. Ambulatory Impedance Cardiography. The Systems and their Applications. Series: Lecture Notes in Electrical Engineering, 2011, Berlin and Heidelberg, Springer-Verlag.

[15] Kubicek WG, Karnegis JN, Patterson RP, Witsoe DA, Mattson RH. Development and evaluation of an impedance cardiac output system. Aerospace Medicine 1966;37:120812.

[16] Ebert TJ, Eckberg DL, Vetrvec GM, Cowley MJ. Impedance cardiograms reliably estimate beat-by-beat changes of left ventricular stroke volume in humans, Cardiovasc Res 1984;18:354-60

[17] Scherhag A, Kaden JJ, Kentschke E, Sueselbeck T, Borggrefe M. Comparison of impedance cardiography and thermodilution-derived measurements of stroke volume and cardiac output at rest and during exercise testing. Cardiovasc Drugs Ther 2005;19(2):141-7.

[18] Cybulski G, Strasz A, Niewiadomski W, Gąsiorowska A. Impedance cardiography: Recent advancements. Cardiol J 2012;19(5):550-6.

[19] Fellahi JL, Fischer MO. Electrical bioimpedance cardiography: an old technology with new hopes for the future. J Cardiothorac Vasc Anesth 2014;28(3):755-60.

[20] Lozano DL, Norman G, Knox D, Wood BL, Miller BD, Emery CF, Berntson GG. Where to $\mathrm{B}$ in $\mathrm{dZ} / \mathrm{dt}$. Psychophysiology 2007;44(1):113-19

Address for correspondence:

Gerard Cybulski

Warsaw University of Technology

Faculty of Mechatronics

Institute of Metrology and Biomedical Engineering

Sw. A. Boboli 8,

02-575 Warsaw

Poland

G.Cybulski@mchtr.pw.edu.pl 